\title{
889.
}

\section{ON A DIFFERENTIAL EQUATION AND THE CONSTRUCTION OF MILNER'S LAMP.}

[From the Proceedings of the Edinburgh Mathematical Society, vol. v. (1887), pp. $99-101$.

WHAT sort of an equation is

$$
b^{3} \cos (\alpha+\theta)=a \cos \theta \int_{\theta}^{\beta} r^{2} d \theta-\frac{2}{3}\left\{\cos \theta \int_{\theta}^{\beta} r^{3} \cos \theta d \theta+\sin \theta \int_{\theta}^{\beta} r^{3} \sin \theta d \theta\right\} ?
$$

Write

$$
X=\int_{\theta}^{\beta} r^{2} d \theta, \quad Y=\int_{\theta}^{\beta} r^{3} \cos \theta d \theta, \quad Z=\int_{\theta}^{\beta} r^{3} \sin \theta d \theta
$$

and start with the equations

$$
\begin{gathered}
d \theta=\frac{d X}{-r^{2}}=\frac{d Y}{-r^{3} \cos \theta}=\frac{d Z}{-r^{3} \sin \theta} \cdots \cdots \cdots \\
\left(\frac{d^{2}}{d \theta^{2}}+1\right)\left\{a \cos \theta \cdot X-\frac{2}{3}(Y \cos \theta+Z \sin \theta)\right\}=0
\end{gathered}
$$

This last gives

$$
(r-a \cos \theta) d r+a r \sin \theta \cdot d \theta=0
$$

and the system thus is

$$
d \theta=\frac{d X}{-r^{2}}=\frac{d Y}{-r^{3} \cos \theta}=\frac{d Z}{-r^{3} \sin \theta}=\frac{(r-a \cos \theta) d r}{-a r \sin \theta}
$$

viz. this is a system of ordinary differential equations between the five variables $\theta$, $r, X, Y, Z$ : the system can therefore be integrated with four arbitrary constants, and these may be so determined that for the value $\beta$ of $\theta, X, Y, Z$ shall be each $=0$; and $r$ shall have the value $r_{0}$. 
But this being so, from the assumed equations (3) and (4) we have

$$
X=\int_{\theta}^{\beta} r^{2} d \theta, \quad Y=\int_{\theta}^{\beta} r^{3} \cos \theta d \theta, \quad Z=\int_{\theta}^{\beta} r^{3} \sin \theta d \theta
$$

and further, by integration of (4),

$$
L \cos \theta+M \sin \theta=a \cos \theta \cdot X-\frac{2}{3}(Y \cos \theta+Z \sin \theta) .
$$

Here $L$ and $M$ denote properly determined constants: viz. the conclusion is that $r, X, Y, Z$ admit of being determined as functions of $\theta$ and of an arbitrary constant $r_{0}$, in such wise that

$$
a \cos \theta \cdot X-\frac{2}{3}(Y \cos \theta+Z \sin \theta)
$$

shall be a function of $\theta$, of the proper form $L \cos \theta+M \sin \theta$, but not so that it shall be the precise function $b^{3} \cos (\alpha+\theta)$. To make it have this value, we must have $L=b^{3} \cos \alpha, M=-b^{3} \sin \alpha$ (where $L, M$ are given functions of $a, \beta, r_{0}$ ), i.e. we must have two given relations between $a, b, \alpha, \beta, r_{0}$ : or treating $r_{0}$ as a disposable constant, we must have one given relation between $a, b, \alpha, \beta$.

The equation $d \theta=\frac{r-a \cos \theta}{-a r \sin \theta} d r$ gives $r^{2}-2 a r \cos \theta=C$, where $C=r_{0}^{2}-2 a r_{0} \cos \beta$. There would be considerable difficulty in working the question out with $r_{0}$ arbitrary, but we may do it easily enough for the particular value $r_{0}=0$ or $r_{0}=2 a \cos \beta$, giving $C=0$ and therefore $r=2 a \cos \theta$ : and we ought in this case to be able to satisfy the given equation not in general but with two determinate relations between the constants $a, b, \alpha, \beta$.

We have

$$
\begin{aligned}
& \int \cos ^{2} \theta d \theta=\frac{1}{2} \theta+\frac{1}{4} \sin 2 \theta \\
& \int \cos ^{4} \theta d \theta=\frac{3}{8} \theta+\frac{1}{4} \sin 2 \theta+\frac{1}{32} \sin 4 \theta \\
& \int \cos ^{3} \theta \sin \theta d \theta=-\frac{1}{4} \cos ^{4} \theta
\end{aligned}
$$

And thence

$$
\begin{aligned}
a \cos & \theta \cdot X-\frac{2}{3}(Y \cos \theta+Z \sin \theta) \\
= & 4 a^{3} \cos \theta\left\{\frac{1}{2}(\beta-\theta)+\frac{1}{4}(\sin 2 \beta-\sin 2 \theta)\right\} \\
& -\frac{16}{3} a^{3} \cos \theta\left\{\frac{3}{8}(\beta-\theta)+\frac{1}{4}(\sin 2 \beta-\sin 2 \theta)+\frac{1}{32}(\sin 4 \beta-\sin 4 \theta)\right\} \\
& -\frac{16}{3} a^{3} \sin \theta\{ \\
= & -\frac{1}{3} a^{3} \cos \theta(\sin 2 \beta-\sin 2 \theta) \\
& -\frac{1}{6} a^{3} \cos \theta(\sin 4 \beta-\sin 4 \theta) \\
& +\frac{4}{3} a^{3} \sin \theta\left(\cos ^{4} \beta-\cos ^{4} \theta\right),
\end{aligned}
$$


where the terms containing $\beta$ are readily reduced to $\frac{4}{3} a^{3} \cos ^{3} \beta \sin (\theta-\beta)$; hence also the terms without $\beta$ disappear of themselves: and we have

$$
a \cos \theta \cdot X-\frac{2}{3}(Y \cos \theta+Z \sin \theta)=\frac{4}{3} a^{3} \cos ^{3} \beta \cdot \sin (\theta-\beta)
$$

which may be put

$$
=b^{3} \cos (\theta+\alpha):
$$

viz. this will be so if we have the two relations

$$
\alpha=\frac{1}{2} \pi-\beta ; \text { and } b^{3}=-\frac{4}{3} a^{3} \cos ^{3} \beta .
$$

I make (see figure) Milner's lamp, with a circular section, $\beta$ arbitrary, but a

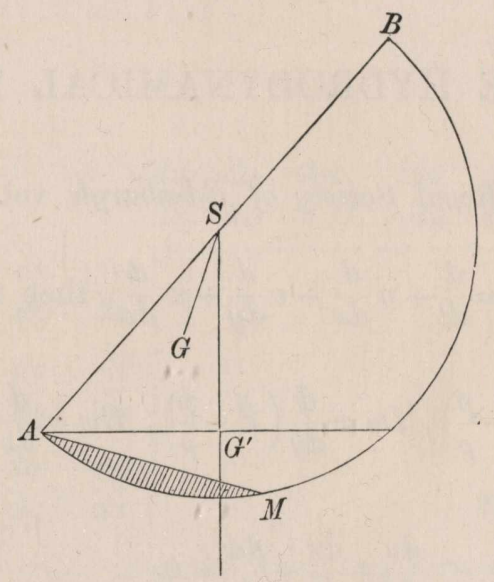

segment $A M(\angle S A M=\beta)$ made solid. $G$ in the line $S G$ at right angles to $A M$ is the 'c. G. of the lamp, and $G^{\prime}$ the c. G. of the oil.

And this seems to be the only form-for the pole of $r$ must, it seems to me, be on the bounding circle-viz. in the equation $r^{2}-2 a r \cos \theta=C$, we must have $C=0$. 